\title{
Eigenvalues of a Differential Operator and Zeros of the Riemann $\zeta$-Function
}

\author{
Liming $\mathrm{Ge}^{1}$, Xian-Jin $\mathrm{Li}^{2}$, Dongsheng $\mathrm{Wu}^{1,2, *}$ and Boqing $\mathrm{Xue}^{3}$ \\ ${ }^{1}$ Academy of Mathematics and Systems Science, Chinese Academy of Sciences, \\ Beijing 100190, China \\ 2 Department of Mathematics, Brigham Young University Provo, Utah 84602, USA \\ ${ }^{3}$ Institute of Mathematical Sciences, ShanghaiTech University, Shanghai 201210, \\ China
}

Received 6 August 2019; Accepted (in revised version) 21 October 2019

Dedicated to Professor Weiyi Su on the occasion of her 80th birthday

\begin{abstract}
The eigenvalues of a differential operator on a Hilbert-Pólya space are determined. It is shown that these eigenvalues are exactly the nontrivial zeros of the Riemann $\zeta$-function. Moreover, their corresponding multiplicities are the same.
\end{abstract}

Key Words: Hilbert-Pólya space, zeros of zeta function, differential operator, eigenvalue.

AMS Subject Classifications: 11M06, 11Q20, 46A15

\section{Introduction and preliminaries}

In studying the Riemann hypothesis it is important to establish a connection between spectrum of operators and zeros of the Riemann zeta function. This is our continued effort for finding such an interplay.

Let $\mathcal{S}(\mathbb{R})$ be the Schwartz space on $\mathbb{R}$. Functions in $\mathcal{S}(\mathbb{R})$ are smooth and rapid decreasing when $|x| \rightarrow \infty$. That is,

$$
\mathcal{S}(\mathbb{R})=\left\{f \in C^{\infty}(\mathbb{R})\left|\sup _{x \in \mathbb{R}}\right| x^{m} f^{(n)}(x) \mid<\infty \text { for } m, n \in \mathbb{N}\right\},
$$

where $\mathbb{N}=\{0,1, \cdots\}$. Denote by $\mathcal{H}_{\cap}$ the subspace of all even functions $f$ in $\mathcal{S}(\mathbb{R})$ with $f(0)=\mathfrak{F} f(0)=0$. Here

$$
\mathfrak{F} f(x)=\int_{-\infty}^{\infty} f(y) e^{-2 \pi i x y} d y
$$

\footnotetext{
${ }^{*}$ Corresponding author. Email addresses: liming@math.ac.cn (L. Ge), xianjin@byu.edu (X. Li), wu2017fall@ mathematics.byu.edu (D. Wu), xuebq@shanghaitech.edu.cn (B. Xue) 
is the Fourier transform of $f$. In this paper, we may identify functions in $\mathcal{H}_{\cap}$ with their restrictions on $(0, \infty)$.

Denote by $\mathcal{H}_{-}$the set of all smooth functions on $(0, \infty)$ that decrease rapidly when $x \rightarrow 0+$ and $\infty$. Specifically,

$$
\mathcal{H}_{-}=\left\{f \in C^{\infty}(0, \infty)\left|\sup _{x>0}\right| x^{m} f^{(n)}(x) \mid<\infty \text { for }|m|, n \in \mathbb{N}\right\} .
$$

For $f \in \mathcal{H}_{\cap}$ we denote by $Z$ the action given by

$$
Z f(x)=\sum_{n=1}^{\infty} f(n x), \quad x>0 .
$$

By Poisson's summation formula, $Z \mathcal{H}_{\cap} \subseteq \mathcal{H}_{-}$. The quotient space $\mathcal{H}_{-} / Z \mathcal{H}_{\cap}$, denoted by $\mathcal{H}$, is regarded as a Hilbert-Pólya space, although such a quotient does not carry a Hilbert space structure.

The fundamental differential operator $D$ on $\mathcal{H}_{-}$is given by

$$
D f(x)=-x f^{\prime}(x), \quad f \in \mathcal{H}_{-} .
$$

Notice that $D \mathcal{H}_{-} \subseteq \mathcal{H}_{-}$and $D\left(Z \mathcal{H}_{\cap}\right) \subseteq Z \mathcal{H}_{\cap}$. The operator $D$ thus induces a differential operator on $\mathcal{H}$, which we denote by $D_{-}$. We follow the notation introduced in [5]. For basics on the Riemann zeta function $\zeta(s)$, we refer to [6].

The Hilbert-Pólya conjecture states that the non-trivial zeros of the Riemann $\zeta$ function correspond (in certain canonical way) to the eigenvalues of some operator, and Riemann Hypothesis is equivalent to the self-adjointness of the operator.

In the direction of the Hilbert-Pólya conjecture, a spectral interpretation for zeros on the critical line $\operatorname{Re}(s)=\frac{1}{2}$ was given by A. Connes [1]. He constructed a closed, densely defined, unbounded differential operator $D_{\chi}$ and a Hilbert-Pólya space $\mathcal{H}_{\chi}$. His operator $D_{\chi}$ has discrete spectrum, which is the set of imaginary parts of critical zeros of the $L$ function with Grössencharacter $\chi$ [1, Theorem 1, pp. 40].

In [2], L. Ge studied the action of the differential operator $D$ on a Hilbert-Pólya space, which is slightly different from $\mathcal{H}$, and obtained that the point spectrum of $D$ coincides with the nontrivial zeros of $\zeta(s)$.

Meyer proved in [5, Corrollary 4.2, pp. 8] that the eigenvalues of the transpose $D_{-}^{t}$ of $D_{-}$(acting on the space of continuous linear functionals on $\mathcal{H}$ ) are exactly the non-trivial zeors of $\zeta(s)$, and the algebraic multiplicity of an eigenvalue is equal to the vanishing order of the corresponding zero of $\zeta(s)$.

$\mathrm{Li}$ [4] proved by an explicit construction that the nontrivial zeros of $\zeta(s)$ are the eigenvalues of $D_{-}$acting on $\mathcal{H}$ (instead of $D_{-}^{t}$ in [5]), which we state as follows (Theorem 1.1 and Theorem 1.4 in [4]).

Theorem 1.1 ([4]). Let $\rho$ be a nontrivial zero of $\zeta(s)$. Let $F_{\rho}$ be the function given by

$$
F_{\rho}(x)=\int_{1}^{\infty} Z \eta(t x) t^{\rho-1} d t,
$$


where

$$
\eta(x)=8 \pi x^{2}\left(\pi x^{2}-\frac{3}{2}\right) e^{-\pi x^{2}} .
$$

Then $F_{\rho}$ is an eigenfunction of $D_{-}$on $\mathcal{H}$ associated with eigenvalue $\rho$. Moreover, the eigenvalue $\rho$ has geometric multiplicity one.

A question is that whether or not every eigenvalue of $D_{-}$on $\mathcal{H}$ is a nontrivial zero of the Riemann zeta function. In this paper, we give an affirmative answer to the question by proving that there is a one-to-one correspondence between the set of eigenvalues of $D_{-}$acting on $\mathcal{H}$ and the set of nontrivial zeros of $\zeta(s)$. Moreover, we also show that the algebraic multiplicity of any eigenvalue of $D_{-}$is equal to the vanishing order of the corresponding zero of $\zeta(s)$. Recall that the algebraic multiplicity of an eigenvalue $\lambda$ of an operator $T$ is the dimension of the space $\bigcup_{n>1} \operatorname{ker}(T-\lambda)^{n}$. Some of these results are hinted in [5]. The goal of this paper is to give a detailed proof of our main result which we state as the following theorem.

Theorem 1.2. A complex number $\rho$ is an eigenvalue of $D_{-}$on $\mathcal{H}$ if and only if $\rho$ is a nontrivial zero of $\zeta(s)$. Moreover, its algebraic multiplicity is equal to the vanishing order of $\zeta(s)$ at $\rho$.

The proof of Theorem 1.2 will be presented in Section 3. In Section 2, we study the eigenvalues of $D_{-}$and will show that they have a similar distribution as the nontrivial zeros of $\zeta(s)$, which is stated as follows.

Theorem 1.3. If $\lambda$ is an eigenvalue of $D_{-}$on $\mathcal{H}$, then $0<\operatorname{Re}(\lambda)<1$.

Now we give the detailed proof of this theorem in the following section.

\section{Eigenvalues of $D_{-}$}

Let $f$ be a measurable function on $(0, \infty)$. Denote by

$$
\widehat{f}(s)=\int_{0}^{\infty} f(x) x^{s-1} d x, \quad s \in \mathbb{C},
$$

the Mellin transform of $f$, provided that the above integral exists for some $s$. For $f \in \mathcal{H}_{-}$, it is not hard to check that $\widehat{f}(s)$ is entire on $\mathbb{C}$ and, from integration by parts,

$$
\widehat{D f}(s)=-\int_{0}^{\infty} x f^{\prime}(x) x^{s-1} d x=-\int_{0}^{\infty} x^{s} d f(x)=s \int_{0}^{\infty} f(x) x^{s-1} d x=s \widehat{f}(s) .
$$

Let $g$ be a function in $\mathcal{H}_{\cap}$. Since $g(x)$ decreases rapidly as $x \rightarrow \infty$ and $g$ is continuous at 0 , the integral

$$
\int_{0}^{\infty} g(x) x^{s-1} d x
$$


exists when $\operatorname{Re}(s)>0$, and we have

$$
\widehat{\mathrm{Zg}}(s)=\int_{0}^{\infty}\left(\sum_{n=1}^{\infty} g(n x)\right) x^{s-1} d x=\sum_{n=1}^{\infty} \int_{0}^{\infty} g(y)\left(\frac{y}{n}\right)^{s-1} d\left(\frac{y}{n}\right)=\zeta(s) \widehat{g}(s)
$$

when $\operatorname{Re}(s)>1$. Now we have some key properties of the operators $D$ and $Z$. We need to introduce another operator $J$.

Let $f$ be a function in $\mathcal{H}_{-}$. Denote by $J$ the operator given by $J f(x)=x^{-1} f\left(x^{-1}\right)$. It is easy to see that $J \mathcal{H}_{-}=\mathcal{H}_{-}$and $\widehat{J f}(s)=\widehat{f}(1-s)$. The operator J plays an important role in the symmetry of $\mathbb{C}$ along the critical line $\operatorname{Re}(s)=1 / 2$ and induces a unitary operator from $L^{2}(0,1)$ onto $L^{2}(1, \infty)$ (see [3]). Here we are interested in its connections with operators $Z, \mathfrak{F}$ and the Mellin transform.

For $g \in \mathcal{H}_{\cap}$, we have $\mathfrak{F} g \in \mathcal{H}_{\cap}$. Applying the Poisson summation formula to $g \in \mathcal{H}_{\cap}$, since both $g$ and $\mathfrak{F} g$ are even functions vanishing at zero, we have

$$
J Z g(x)=\frac{1}{x} \sum_{n=1}^{\infty} g\left(\frac{n}{x}\right)=\sum_{n=1}^{\infty} \mathfrak{F} g(n x)=Z \mathfrak{F} g(x)
$$

Hence

$$
\widehat{J Z g}(s)=\widehat{Z g}(1-s)=\widehat{Z F g}(s) .
$$

So $J Z \mathcal{H}_{\cap} \subseteq Z \mathcal{H}_{\cap}$. Notice that $J^{2}$ is the identity operator, we have

$$
J Z \mathcal{H}_{\cap}=Z \mathcal{H}_{\cap} .
$$

With the above discussion, we are ready to prove the following lemma.

Lemma 2.1. Let $\lambda$ be an eigenvalue of $D_{-}$on $\mathcal{H}$. Then $\bar{\lambda}$ and $1-\lambda$ are both eigenvalues of $D_{-}$ on $\mathcal{H}$.

Proof. For any given $\lambda$, an eigenvalue of $D_{-}$on $\mathcal{H}$, there is a function $f \in \mathcal{H}_{-} \backslash Z \mathcal{H}_{\cap}$ and a $g \in \mathcal{H}_{\cap}$ such that $D f-\lambda f \in Z \mathcal{H}_{\cap}$ and

$$
-x f^{\prime}(x)=\lambda f(x)+Z g(x)
$$

Taking conjugate we have

$$
-x \overline{f^{\prime}(x)}=\bar{\lambda} \overline{f(x)}+\overline{Z g(x)}, \text { i.e., } D \bar{f}=\overline{\lambda f}+Z \bar{g} .
$$

Notice that $\bar{f} \in \mathcal{H}_{-} \backslash Z \mathcal{H}_{\cap}$ and $\bar{g} \in \mathcal{H}_{\cap}$, we see that $\bar{\lambda}$ is an eigenvalue of $D_{-}$on $\mathcal{H}$.

Next we prove that $1-\lambda$ is also an eigenvalue of $D_{-}$on $\mathcal{H}$ by showing that $J f$ is an eigenfunction with eigenvalue $1-\lambda$. Since $J \mathcal{H}_{-}=\mathcal{H}_{-}$and $J Z \mathcal{H}_{\cap}=Z \mathcal{H}_{\cap}$, we have $J f \in \mathcal{H}_{-} \backslash Z \mathcal{H}_{\cap}$. Applying the Mellin transform to (2.2), we have

$$
s \widehat{f}(s)=\lambda \widehat{f}(s)+\zeta(s) \widehat{g}(s) .
$$


Replacing $s$ by $1-s$ and applying (2.1), we obtain

$$
\begin{aligned}
& (1-s) \widehat{f}(1-s)=\lambda \widehat{f}(1-s)+\zeta(1-s) \widehat{g}(1-s) \\
= & \lambda \widehat{f}(1-s)+\widehat{J Z g}(s)=\lambda \widehat{f}(1-s)+\widehat{Z \mathfrak{F} g}(s) .
\end{aligned}
$$

This formula implies that

$$
s \widehat{f}(1-s)=(1-\lambda) \widehat{f}(1-s)-\zeta(s) \widehat{\mathfrak{F g}}(s) .
$$

Notice that $\widehat{J f}(s)=\widehat{f}(1-s)$, we thus have

$$
s \widehat{J f}(s)=(1-\lambda) \widehat{J f}(s)-\zeta(s) \widehat{\mathfrak{F g}}(s) .
$$

Since $J f \in \mathcal{H}_{-} \backslash Z \mathcal{H}_{\cap}$ and $\mathfrak{F g} \in \mathcal{H}_{\cap}$, applying the Mellin inverse transform we obtain

$$
-x(J f)^{\prime}(x)=(1-\lambda) J f(x)+Z(-\mathfrak{F g})(x) .
$$

Hence $1-\lambda$ is also an eigenvalue of $D_{-}$on $\mathcal{H}$. This completes the proof of the lemma.

We already see that the integral $\int_{0}^{\infty} g(x) x^{s-1} d x$ exists when $\operatorname{Re}(s)>0$, where $g$ is a function in $\mathcal{H}_{\cap}$. In fact, we can show that the above integral exists when $\operatorname{Re}(s)>-2$. Since $g$ is an even Schwartz function, the derivative $g^{\prime}$ is odd which implies that $g^{\prime}(0)=0$. Let

$$
c=\sup _{|x| \leq 1}\left|g^{\prime \prime}(x)\right|
$$

be the maximum of $g^{\prime \prime}$ on $[-1,1]$. For $x \in[-1,1]$, we have, by Mean Value Theorem,

$$
|g(x)-g(0)|=|g(x)| \leq\left|g^{\prime}(\xi) x\right|=\left|g^{\prime}(\xi)-g^{\prime}(0)\right||x| \leq\left|g^{\prime \prime}\left(\xi^{\prime}\right) x\right||x| \leq c x^{2},
$$

where $-1<\xi, \xi^{\prime}<1$. Hence for $s \in \mathbb{C}$ with $\operatorname{Re}(s)>-2$, the integral $\int_{0}^{1} g(x) x^{s-1} d x$ exists. While $\int_{1}^{\infty} g(x) x^{s-1} d x$ is well defined for any $s \in \mathbb{C}, \int_{0}^{\infty} g(x) x^{s-1} d x$ exists when $\operatorname{Re}(s)>-2$.

When $g$ is not even and $g \in \mathcal{S}(\mathbb{R})$, we do not know the behavior of $g(x)$ near $x=0$. But we can show that $\widehat{g}$ admits a meromorphic continuation on $\mathbb{C}$. In fact, from integration by parts,

$$
\int_{0}^{\infty} g(x) x^{s-1} d x=\left.\frac{x^{s}}{s} g(x)\right|_{0} ^{\infty}-\frac{1}{s} \int_{0}^{\infty} g^{\prime}(x) x^{s} d x=-\frac{1}{s} \int_{0}^{\infty} g^{\prime}(x) x^{s} d x
$$

for $s \in \mathbb{C}$ with $\operatorname{Re}(s)>0$. Since $g \in \mathcal{S}(\mathbb{R})$, we have $g^{\prime} \in \mathcal{S}(\mathbb{R})$. So the last integral exists for $\operatorname{Re}(s)>-1$. Hence we obtain a meromorphic continuation of $\widehat{g}$ on $\{s \in \mathbb{C} \mid \operatorname{Re}(s)>-1\}$. Repeating this procedure, we obtain a meromorphic continuation of $\widehat{g}$ to the complex plane $\mathbb{C}$. Such technique can be applied to the extension of the Mellin transform of more general functions.

To prove Theorem 1.3, we need to establish a lemma on functions in $\mathcal{H}_{\cap}$. 
Lemma 2.2. Assume $g \in \mathcal{H}_{\cap}$. Suppose that $\widehat{g}\left(s_{0}\right)=0$ for some $s_{0} \in \mathbb{C}$ with $\operatorname{Re}\left(s_{0}\right)>-1$ and $\operatorname{Re}\left(s_{0}\right) \neq 1$. Define, for $x \in \mathbb{R}$,

$$
f(x)=\int_{1}^{\infty} g(t x) t^{s_{0}-1} d t
$$

Then we have

i) $f(x)=\int_{1}^{\infty} g(t x) t^{s_{0}-1} d t=-\int_{0}^{1} g(t x) t^{s_{0}-1} d t ;$

ii) $f \in \mathcal{H}_{\cap}$; and

iii) $\widehat{g}(s)=\widehat{f}(s)\left(s-s_{0}\right)$.

Proof. First we prove i). Notice that $|g(x)| \leq c|x|$ for $c=\sup _{x \in \mathbb{R}}\left|g^{\prime}(x)\right|<\infty$. The integral $\int_{0}^{\infty} g(t) t^{s-1}$ exists when $\operatorname{Re}(s)>-1$. Hence we only need to show that

$$
\int_{0}^{\infty} g(t x) t^{s_{0}-1} d t=0
$$

holds for any $x \in \mathbb{R}$.

Note that

$$
\int_{0}^{\infty} g(t) t^{s_{0}-1} d t=\widehat{g}\left(s_{0}\right)=0 .
$$

For any given $x>0$, substituting $t$ by $t x$ in the above equation, we have

$$
x^{s_{0}} \int_{0}^{\infty} g(t x) t^{s_{0}-1} d t=\int_{0}^{\infty} g(t) t^{s_{0}-1} d t=\widehat{g}\left(s_{0}\right)=0 .
$$

So

$$
\int_{0}^{\infty} g(t x) t^{s_{0}-1} d t=0
$$

for $x>0$. For $x<0$, since $g$ is even, we also have

$$
\int_{0}^{\infty} g(t x) t^{s_{0}-1} d t=\int_{0}^{\infty} g(-t x) t^{s_{0}-1} d t=0 .
$$

For $x=0$, we have

$$
\int_{0}^{\infty} g(t \cdot 0) t^{s_{0}-1} d t=\int_{0}^{\infty} g(0) t^{s_{0}-1} d t=0
$$

as $g(0)=0$. Hence

$$
\int_{0}^{\infty} g(t x) t^{s_{0}-1} d t=0
$$


for $x \in \mathbb{R}$. Therefore,

$$
f(x)=\int_{1}^{\infty} g(t x) t^{s_{0}-1} d t=-\int_{0}^{1} g(t x) t^{s_{0}-1} d t .
$$

Next we verify ii). It is clear that $f$ is an even function. First we check the smoothness of $f$. Since $g$ is a Schwartz function, by Lebesgue's Dominated Convergence Theorem, we see that $f$ is smooth on $\mathbb{R} \backslash\{0\}$ and, for $x \neq 0, n \in \mathbb{N}$,

$$
f^{(n)}(x)=\int_{1}^{\infty} g^{(n)}(t x) t^{n+s_{0}-1} d t=-\int_{0}^{1} g^{(n)}(t x) t^{n+s_{0}-1} d t .
$$

It remains to show that $f$ is smooth at 0 . We start with the continuity of $f$ at 0 . Since $|g(x)| \leq c|x|$ for $x \in[0,1]$, we have

$$
|f(x)| \leq c \int_{0}^{1} t x t^{\operatorname{Re}\left(s_{0}\right)-1} d t \leq c x .
$$

Hence

$$
\lim _{x \rightarrow 0} f(x)=0=f(0) .
$$

Next we prove inductively that $f^{(n)}(0)$ exists for $n \geq 1$. Notice that, by Mean Value Theorem, the function $\frac{g(t)-g(0)}{t}$ is bounded by $\sup _{x \in \mathbb{R}}\left|g^{\prime}(x)\right|$ for $t \neq 0$. Since $\operatorname{Re}\left(s_{0}\right)>-1$, we have, by Lebesgue's dominated convergence theorem,

$$
\begin{aligned}
f^{\prime}(0) & =\lim _{x \rightarrow 0} \frac{-\int_{0}^{1} g(t x) t^{s_{0}-1} d t+\int_{0}^{1} g(0) t^{s_{0}-1} d t}{x} \\
& =\lim _{x \rightarrow 0}-\int_{0}^{1} \frac{g(t x)-g(0)}{t x} t^{s_{0}} d t=-\int_{0}^{1} g^{\prime}(0) t^{s_{0}} d t .
\end{aligned}
$$

Assume that

$$
f^{(k)}(0)=-\int_{0}^{1} g^{(k)}(0) t^{k+s_{0}-1} d t
$$

for some $k \geq 1$. Notice that the function $\frac{g^{(k)}(t)-g^{(k)}(0)}{t}$ is bounded by $\sup _{x \in \mathbb{R}}\left|g^{k+1}(x)\right|$ for $t \neq 0$. For $n=k+1$, we apply the above equation (2.3), use Lebesgue's Dominated Convergence Theorem, and then obtain

$$
\begin{aligned}
& f^{(k+1)}(0)=\lim _{x \rightarrow 0} \frac{-\int_{0}^{1} g^{(k)}(t x) t^{k+s_{0}-1} d t+\int_{0}^{1} g^{(k)}(0) t^{k+s_{0}-1} d t}{x} \\
= & \lim _{x \rightarrow 0}-\int_{0}^{1} \frac{g^{(k)}(t x)-g^{(k)}(0)}{t x} t^{k+s_{0}} d t=-\int_{0}^{1} g^{(k+1)}(0) t^{k+s_{0}} d t .
\end{aligned}
$$

Thus, (2.3) holds for all $k \in \mathbb{N}$. Hence $f$ is smooth on $\mathbb{R}$. 
Now we prove that $f(x)$ decays rapidly as $|x| \rightarrow \infty$, it suffices to check that $x^{m} f^{(n)}(x) \rightarrow 0$ when $|x| \rightarrow \infty$ for any $m, n \in \mathbb{N}$. Since $g$ is a Schwartz function, there is a constant $C>0$ such that

$$
\begin{aligned}
\left|x^{m} f^{(n)}(x)\right| & =\left|x^{m} \int_{1}^{\infty} g^{m}(t x) t^{n+s_{0}-1} d t\right| \\
& \leq C\left|\int_{1}^{\infty} x^{m}(t x)^{-m-1-\operatorname{Re}\left(s_{0}\right)} t^{n+s_{0}-1} d t\right| \\
& \leq \frac{C}{|x|} \int_{1}^{\infty} t^{-2} d t=\frac{C}{|x|} \rightarrow 0, \quad|x| \rightarrow \infty .
\end{aligned}
$$

Hence $f \in \mathcal{S}(\mathbb{R})$.

In the above, we have seen that $f(0)=0$. It remains to show that $\mathfrak{F} f(0)=0$. If $-1<\operatorname{Re}\left(s_{0}\right)<1$, then there is a $\delta$ such that $0<\delta<1$ and $\operatorname{Re}\left(s_{0}\right)<\delta$. Thus we have

$$
\begin{aligned}
|\mathfrak{F} f(0)| & =\left|\int_{-\infty}^{\infty} f(x) d x\right|=\left|\int_{-\infty}^{\infty} \int_{1}^{\infty} g(t x) t^{s_{0}-1} d t d x\right| \\
& \leq \int_{|x|<1} \int_{1}^{\infty}|g(t x)| t^{\operatorname{Re}\left(s_{0}\right)-1} d t d x+\int_{|x| \geq 1} \int_{1}^{\infty}|g(t x)| t^{\operatorname{Re}\left(s_{0}\right)-1} d t d x \\
& \leq \int_{|x|<1} \int_{1}^{\infty} C_{1}(t x)^{-\delta} t^{\operatorname{Re}\left(s_{0}\right)-1} d x d t+\int_{|x| \geq 1} \int_{1}^{\infty} C_{2}(t x)^{-2} t^{\operatorname{Re}\left(s_{0}\right)-1} d x d t
\end{aligned}
$$

for some constants $C_{1}, C_{2}>0$. So the double integral $\int_{-\infty}^{\infty} \int_{1}^{\infty} g(t x) t^{s_{0}-1} d t d x$ is absolutely integrable. By Fubini's Theorem,

$$
\mathfrak{F} f(0)=\int_{1}^{\infty} t^{s_{0}-1} \int_{-\infty}^{\infty} g(t x) d x d t=\int_{1}^{\infty} t^{s_{0}-2} \int_{-\infty}^{\infty} g(x) d x d t=0 .
$$

If $\operatorname{Re}\left(s_{0}\right)>1$, then we can choose $\delta^{\prime}$ such that $\delta^{\prime}>1$ and $\operatorname{Re}\left(s_{0}\right)-1-\delta^{\prime}>-1$. Then we have

$$
\begin{aligned}
|\mathfrak{F} f(0)| & =\left|\int_{-\infty}^{\infty} f(x) d x\right|=\left|\int_{-\infty}^{\infty} \int_{0}^{1} g(t x) t^{s_{0}-1} d t d x\right| \\
& \leq \int_{|x|<1} \int_{0}^{1}|g(t x)| t^{\operatorname{Re}\left(s_{0}\right)-1} d t d x+\int_{|x| \geq 1} \int_{0}^{1}|g(t x)| t^{\operatorname{Re}\left(s_{0}\right)-1} d t d x \\
& \leq \int_{|x|<1} \int_{0}^{1} C_{3} t^{\operatorname{Re}\left(s_{0}\right)-1} d x d t+\int_{|x| \geq 1} \int_{0}^{1} C_{4}(t x)^{-\delta^{\prime}} t^{\operatorname{Re}\left(s_{0}\right)-1} d x d t
\end{aligned}
$$

for some constants $C_{3}, C_{4}>0$. Again, by Fubini's Theorem,

$$
\mathfrak{F} f(0)=-\int_{0}^{1} t^{s_{0}-1} \int_{-\infty}^{\infty} g(t x) d x d t=-\int_{0}^{1} t^{s_{0}-2} \int_{-\infty}^{\infty} g(x) d x d t=0 .
$$

Therefore $f \in \mathcal{H}_{\cap}$. 
Finally, we prove statement iii). By partial integration we have

$$
-x f^{\prime}(x)=\int_{1}^{\infty}-x g^{\prime}(t x) t^{s_{0}} d t=-\left.g(t x) t^{s_{0}}\right|_{1} ^{\infty}+s_{0} \int_{1}^{\infty} g(t x) t^{s_{0}-1} d t=g(x)+s_{0} f(x) .
$$

Applying the Mellin transform, we obtain that $s \widehat{f}(s)=\widehat{g}(s)+s_{0} \widehat{f}(s)$. Hence $\widehat{g}(s)=$ $\widehat{f}(s)\left(s-s_{0}\right)$. This completes the proof of the lemma.

Now we are ready to prove Theorem 1.3.

Proof. By Lemma 2.1, it suffices to show that $D_{-}$has no eigenvalue $\lambda$ on $\mathcal{H}$ with $\operatorname{Re}(\lambda)>$ 1 or $\operatorname{Re}(\lambda)=0$.

Assume on the contrary that such $\lambda$ exists, i.e., $\lambda$ is an eigenvalue of $D_{-}$on $\mathcal{H}$ satisfying $\operatorname{Re}(\lambda)>1$ or $\operatorname{Re}(\lambda)=0$. Then there is a function $f \in \mathcal{H}_{-} \backslash Z \mathcal{H}_{\cap}$ and a $g \in \mathcal{H}_{\cap}$ such that $-x f^{\prime}(x)=\lambda f(x)+Z g(x)$. Applying the Mellin transform, we have $s \widehat{f}(s)=\lambda \widehat{f}(s)+\zeta(s) \widehat{g}(s)$. Hence

$$
\frac{\widehat{f}(s)}{\zeta(s)}=\frac{\widehat{g}(s)}{s-\lambda}
$$

By the assumption that $\lambda$ satisfies $\operatorname{Re}(\lambda)=0$ or $\operatorname{Re}(\lambda)>1$, we have $\zeta(\lambda) \neq 0$. So $\widehat{f}(s) / \zeta(s)$ is analytic at $\lambda$. Hence we have $\widehat{g}(\lambda)=0$. By Lemma 2.2 iii), there is a function $g_{1} \in \mathcal{H}_{\cap}$ such that $\widehat{g}(s)=\widehat{g_{1}}(s)\left(s-s_{0}\right)$. Thus $\widehat{f}(s)=\zeta(s) \widehat{g_{1}}(s)$, which implies that $f=Z g_{1} \in Z \mathcal{H}_{\cap}$. This contradicts the fact that $f \in \mathcal{H}_{-} \backslash Z \mathcal{H}_{\cap}$. Hence $D_{-}$has no eigenvalue $\lambda$ with $\operatorname{Re}(\lambda)=0$ or $\operatorname{Re}(\lambda)>1$. This completes the proof of Theorem 1.3.

We may summarize the argument in the proof of Theorem 1.3 and obtain the following corollary. We will apply this corollary several times in the next section.

Corollary 2.1. Let $\lambda$ be a complex number with $\operatorname{Re}(\lambda)>-1$ and $\operatorname{Re}(\lambda) \neq 1$. Assume that $f \in \mathcal{H}_{-} \backslash Z \mathcal{H}_{\cap}$ and $g \in \mathcal{H}_{\cap}$ satisfy $-x f^{\prime}(x)=\lambda f(x)+Z g(x)$ for $x>0$. Then $\widehat{g}(\lambda) \neq 0$ and $\widehat{f}(s) / \zeta(s)$ has a simple pole at $\lambda$, which implies that $\lambda$ is a nontrivial zero of the Riemann zeta function.

\section{Proof of Theorem 1.2}

We first prove a technical lemma.

Lemma 3.1. Let $G$ be an entire function on $\mathbb{C}$. Then $G$ is the Mellin transform of some function in $\mathcal{H}_{-}$if and only if, for every $m \in \mathbb{N}, s^{m} G(s)$ is bounded in any vertical strip with finite width.

Proof. Let $S=\{\sigma+i t \mid \alpha \leq \sigma \leq \beta, t \in \mathbb{R}\}$ be a vertical strip with finite width $\beta-\alpha$, where $-\infty<\alpha<\beta<\infty$. We first show that if $G=\widehat{g}$ for some $g \in \mathcal{H}_{-}$, then for every 
$m \in \mathbb{N}, s^{m} G(s)$ is bounded in any vertical strip with finite width. Since $g \in \mathcal{H}_{-}$, for any $s \in S$, we have

$$
\begin{aligned}
|\widehat{g}(s)| & =\left|\int_{0}^{\infty} g(x) x^{s-1} d x\right| \\
& \leq \int_{0}^{1}\left|g(x) x^{s-1}\right| d x+\int_{1}^{\infty}\left|g(x) x^{s-1}\right| d x \\
& \leq \int_{0}^{1}\left|g(x) x^{\alpha}\right| d x+\int_{1}^{\infty}\left|g(x) x^{\beta+1-2}\right| d x \leq C_{1}+C_{2},
\end{aligned}
$$

where

$$
C_{1}=\sup _{0<x<1}\left|g(x) x^{\alpha}\right| \quad \text { and } \quad C_{2}=\sup _{x \geq 1}\left|g(x) x^{\beta+1}\right| .
$$

Hence $\widehat{g}$ is bounded in $S$.

Next we show that the function $s^{m} \widehat{g}(s)$ is bounded in $S$ for $m \in \mathbb{N}_{>0}$. For $s \in S$ with $|\operatorname{Im}(s)| \leq 1, s^{m} \widehat{g}(s)$ is bounded by continuity. For $s \in S$ with $|\operatorname{Im}(s)|>1, \frac{s}{s+j}$ is bounded for $j=0,1, \cdots, m-1$. Thus there is a constant $C>0$ such that

$$
\left|s^{m}\right| \leq C|s(s+1) \cdots(s+m-1)| \text {. }
$$

By partial integration, we have

$$
\begin{aligned}
\left|s^{m} \widehat{g}(s)\right| & \leq C|s(s+1) \cdots(s+m-1) \widehat{g}(s)| \\
& =C\left|s(s+1) \cdots(s+m-1) \int_{0}^{\infty} g(x) x^{s-1} d x\right| \\
& =C\left|\int_{0}^{\infty} g^{(m)}(x) x^{s+m-1} d x\right| .
\end{aligned}
$$

The last integral in the above formula is the Mellin transform of the function $x^{m} g^{(m)}(x)$. Since $g^{(m)} \in \mathcal{H}_{-}$, we have that this integral is bounded in $S$. Hence $s^{m} \widehat{g}^{(m)}(s)$ is bounded in $S$. This shows that $G=\widehat{g}$ satisfies the desired property.

Conversely, suppose that $G$ is an entire function on $\mathbb{C}$ such that for every $m \in \mathbb{N}$, $s^{m} G(s)$ is bounded in any vertical strip with finite width. Let

$$
h(x)=\frac{1}{2 \pi i} \int_{c-i \infty}^{c+i \infty} G(s) x^{-s} d s, \quad x>0,
$$

be the Mellin inverse transform of $G$. The above integral exists for all $c \in \mathbb{R}$ and is independent of $c$. It is enough to show that $h \in \mathcal{H}_{-}$.

Since $G$ decreases rapidly on every vertical line, we have

$$
h^{(n)}(x)=\frac{1}{2 \pi i} \int_{\mathcal{c}-i \infty}^{c+i \infty} G(s) x^{-s-n}(-s)(-s-1) \cdots(-s-n+1) d x
$$


for $n \in \mathbb{N}$. So $h$ is smooth on $(0, \infty)$. Also, for any $k \in \mathbb{Z}$ and $x>0$, we have

$$
\begin{aligned}
\left|x^{k} h^{(n)}(x)\right| & =\left|\frac{1}{2 \pi i} \int_{k-n-i \infty}^{k-n+i \infty} G(s)(-s)(-s-1) \cdots(-s-n+1) x^{k-n-s} d s\right| \\
& \leq \frac{1}{2 \pi} \int_{k-n-i \infty}^{k-n+i \infty}|G(s)(-s)(-s-1) \cdots(-s-n+1)| d s<\infty .
\end{aligned}
$$

Hence $h \in \mathcal{H}_{-}$. This completes the proof of the lemma.

Now we can prove our main theorem.

Proof. We first show that a complex number $\rho$ is an eigenvalue of $D_{-}$on $\mathcal{H}$ if and only if $\rho$ is a nontrivial zero of the Riemann zeta function.

Let $\rho$ be a nontrivial zero of $\zeta(s)$. We see that $\rho$ is an eigenvalue of $D_{-}$by Li's Theorem [4, Therorem 1.1].

Conversely, let $\rho$ be an eigenvalue of $D_{-}$. By Theorem 1.3, we have $0<\operatorname{Re}(\rho)<1$. Then by Corollary 2.1, $\rho$ is a nontrivial zero of $\zeta(s)$. This proves the first part of Theorem 1.2 .

It remains to show that the algebraic multiplicity of the eigenvalue $\rho$ is equal to the vanishing order of $\zeta(s)$ at $\rho$. Suppose that the function $\zeta(s)$ has vanishing order $m_{0}>0$ at $\rho$. We shall show that the algebraic multiplicity of $D_{-}$at $\rho$ is also $m_{0}$.

Let $F_{1} \in \mathcal{H}_{-} \backslash Z \mathcal{H}_{\cap}$ and $g_{1} \in \mathcal{H}_{\cap}$ such that $-x F_{1}^{\prime}(x)=\rho F_{1}(x)+Z g_{1}$. We have, by Proposition 2.1 , that the function $\widehat{F}_{1}(s) / \zeta(s)$ has a simple pole at $\rho$. So $\widehat{F}_{1}(s)$ has vanishing order $m_{0}-1$ at $\rho$.

Notice that for every $m \in \mathbb{N}, s^{m} \widehat{F}_{1}(s)$ is bounded in any vertical strip with finite width. Then the function $H_{i}, 0 \leq i<m_{0}$, given by

$$
H_{i}(s)=\frac{\widehat{F}_{1}(s)}{(s-\rho)^{i}}
$$

also admits the same property. By Lemma 3.1, $H_{i}$ is the Mellin transform of some function $F_{i+1}$ in $\mathcal{H}_{-}$.

To show that the algebraic multiplicity of $D_{-}$at $\rho$ is $m_{0}$, we let, for $n \geq 1$,

$$
V_{n}=\left\{f \in \mathcal{H}_{-} \mid(D-\rho)^{n} f \in Z \mathcal{H}_{\cap}\right\} .
$$

Notice that the dimension of the subspace $\bigcup_{n=1}^{\infty} V_{n} / Z \mathcal{H}_{\cap}$ in $\mathcal{H}$ is equal to the algebraic multiplicity of the eigenvalue $\rho$ of $D_{-}$acting on $\mathcal{H}$. Denote by $W_{n}=V_{n} / Z \mathcal{H}_{\cap}$. To prove our theorem, it is sufficient to show the following formula

$$
\operatorname{dim} W_{n}= \begin{cases}n, & 1 \leq n \leq m_{0} \\ m_{0}, & n>m_{0}\end{cases}
$$

holds. 
Now we prove formula (3.1). First we show that $V_{n}=V_{n-1}$ for $n>m_{0}$, which implies that $W_{n}=W_{n-1}$ for $n \geq m_{0}$. Assume that there is an $f \in V_{n} \backslash V_{n-1}$. We show that, in this case, $n \leq m_{0}$. The assumption $f \in V_{n} \backslash V_{n-1}$ means that $(D-\rho)^{n} f \in Z \mathcal{H}_{\cap}$ and $(D-\rho)^{n-1} f \notin Z \mathcal{H}_{\cap}$. Denote by $f_{1}$ the function $(D-\rho)^{n-1} f$. Then there is a function $g \in \mathcal{H}_{\cap}$ such that $-x f_{1}^{\prime}(x)=\rho f_{1}(x)+Z g(x)$ for $x>0$. By Proposition 2.1, the function $\widehat{f}_{1}(s) / \zeta(s)$ has a simple pole at $\rho$. Since $\zeta(s)$ has vanishing order $m_{0}$ at $\rho$, the vanishing order of the function $\widehat{f}_{1}(s)=(s-\rho)^{n-1} \widehat{f}(s)$ at $\rho$ is $m_{0}-1$. Because $\widehat{f}$ is analytic at $\rho$, we must have $n \leq m_{0}$. Hence $W_{n}=W_{n-1}$ for $n>m_{0}$.

Next we show that $\operatorname{dim} W_{n}=n$ for $1 \leq n \leq m_{0}$. The case $n=1$ follows from Li's Theorem [4, Theorem 1.4]. For $1<n \leq m_{0}$, it is sufficient to show that $\operatorname{dim}\left(V_{n} / V_{n-1}\right)=1$. Recall that $F_{n} \in \mathcal{H}_{-}$is the Mellin inverse transform of $H_{n-1}$. Applying Mellin transform to $(D-\rho)^{n} F_{n}$ and $(D-\rho)^{n-1} F_{n}$ and noticing that $F_{1} \notin Z \mathcal{H}_{\cap}$ but $(D-\rho) F_{1} \in Z \mathcal{H}_{\cap}$, we conclude that $F_{n} \in V_{n} \backslash V_{n-1}$. Hence $\operatorname{dim}\left(V_{n} / V_{n-1}\right) \geq 1$.

Finally, we show that $\operatorname{dim}\left(V_{n} / V_{n-1}\right) \leq 1$. Let $G_{1}$ ans $G_{2}$ be two functions in $V_{n} \backslash V_{n-1}$. Then $(D-\rho)^{n-1} G_{1},(D-\rho)^{n-1} G_{2} \in V_{1} \backslash Z \mathcal{H}_{\cap}$. By Li's Theorem, there is a complex number $\mu$ such that

$$
(D-\rho)^{n-1} G_{1}-\mu(D-\rho)^{n-1} G_{2}=(D-\rho)^{n-1}\left(G_{1}-\mu G_{2}\right) \in Z \mathcal{H}_{\cap} .
$$

So $G_{1}-\mu G_{2} \in V_{n-1}$. Hence $\operatorname{dim}\left(V_{n} / V_{n-1}\right) \leq 1$. Therefore

$$
\operatorname{dim}\left(W_{n} / W_{n-1}\right)=\operatorname{dim}\left(V_{n} / V_{n-1}\right)=1
$$

for $1 \leq n \leq m_{0}$ and (3.1) holds. This completes the proof of the theorem.

\section{Acknowledgements}

Boqing Xue's work is supported by the National Natural Science Foundation of China (Grant No. 11701549).

\section{References}

[1] A. Connes, Trace formula in noncommutative geometry and the zeros of the Riemman zeta function, Selecta Math., 5 (1999), 29-106.

[2] L. Ge, Lectures on "The Riemann zeta function and Connes's noncommutative geometry", 29th Canadian Symposium On Operator Algebras 2001, https : //www .msri . org/workshops /96/schedules/518.

[3] L. Ge, On the Riemann zeta function, I: KS-transform, Acta Math. Sinica (Chinese Series), 62 (2019), 673-686.

[4] Xian-Jin Li, On spectral theory of the Riemann zeta function, Sci. China Math., 62 (2019), 2317-2330.

[5] R. Meyer, A spectral interpretation for the zeros of the Riemann zeta function, Math. Institut, Georg-August-Univ, Göttingen: Seminars Winter Term 2004/2005, 117-137, Univ. Göttingen, Göttingen, 2005.

[6] E. C. Titchmarsh, The Theory of the Riemann Zeta-Function, Second Edition, Oxford, 1986. 